\title{
Role of nanostructured polymers on the improvement of electrical response-based relative humidity sensors
}

\author{
I. Fratoddi ${ }^{\natural}$, A. Bearzotti ${ }^{\ddagger}$, I. Venditti ${ }^{\natural}$, C. Cametti $^{\S}$, M. V. Russo ${ }^{\natural}$ \\ ${ }^{\natural}$ Dipartimento di Chimica, Universita' di Roma "La Sapienza" \\ Piazzale A. Moro 5, I-00185 - Rome (Italy) \\ ${ }^{\ddagger}$ I.I.A. - CNR Roma Area di Ricerca di Montelibretti \\ $\S$ Dipartimento di Fisica, Universita' di Roma "La Sapienza" \\ Piazzale A. Moro 5, I-00185 - Rome (Italy) and INFM CRS-SOFT, Unita' di Roma 1
}

September 29, 2015

\begin{abstract}
Nanostructured polymers, with different morphologies and different spatial organizations, have found, in last few years, a wide range of uses in humidity sensors, as a viable alternative to ceramic or semiconducting materials and to oxides (perovskite) compounds. Their enhanced sensitivity towards external stimuli has made them ideal candidates in the design of humidity sensors.

This is mainly due to the fact that nanostructured polymers, when embedded with different content of water, represent a heterogeneous system whose dielectric and conductometric properties varies over a wide range, resulting in advantageous sensitive material in resistive-type or capacitive-type humidity sensors. The most notable property of nanostructured polymers is their inherent electrical behavior which is closely connected to the presence of heterogeneities at a nano- or micro- scale level.

In the first part of this paper, we discuss and justify, on the basis of the dielectric theory of heterogeneous systems, how the dielectric properties of nanostructured polymers, independently of their chemical nature, could vary as a consequence of a different amount of water adsorption, meeting the basic requirement of any humidity sensor device. In particular, we have analyzed systems with different porosity and different pore interconnections to cover the most part of the structural arrangements and morphologies nanostructured polymers give rise.

In the second part of the paper, we present some remarkable examples of nanostructured polymers employed in the fabrication of humidity
\end{abstract}


sensors based on changes in the electrical properties (permittivity and electrical conductivity) upon exposure to moisture, highlighting the main features that make them suitable for sensors, with specific emphasis to characteristic parameters, such as sensitivity, response time, hysteresis and durability.

\section{Introduction}

Nanostructured polymers [NSPs] belong to the class of nanomaterials which have, in the last few years, provoked great interest and received great attention as they may be appropriately tailored for a large number of biological and technological applications. Mainly due to the small size of the building blocks (of the order of the nanometer scale) and the high surface-to-volume ratio, these materials are expected to demonstrate unique physico-chemical properties and are promising for a variety of applications including drug and gene delivery [1], tissue engineering [2], biosensors [3], organic electronics and photonics [4], optical devices [5], catalysis [6] and solar cells [7].

This paper gives a look on the use of nanostructured polymers sensing applications devoted to the measurement of the relative humidity $[\mathrm{RH}]$. Our effort is to justify, on the basis of the dielectric theories developed so far for the treatment of highly heterogeneous systems, why nanostructured polymers, whatsoever their chemical properties are and whatsoever the structural conformation they assume, could represent a particularly suitable material to be employed as sensitive element in humidity sensor technologies.

We will do this, considering the most frequently used models dealing with polymer-water mixtures and showing how the dielectric response (characterized by their electrical conductivity and electrical permittivity) covers a wide range of values, directly related to the total amount of water adsorbed.

This paper is organized as follows. It begins with a brief list of humidity definitions and then we discuss the different theoretical models, in the light of the heterogeneous system approximation theory, how nanostructured polymers represent a suitable material for humidity measurements. Finally, we conclude by describing the main characteristics of sensors formulated with differently organized polymeric nanomaterials. Nanostructured polymers employed as sensitive element in the sensors selected here in this certainly not exhaustive list derive from different chemical materials on the basis of different synthesis procedures, are differently organized from a structural, and spatial too, point of view and, moreover, interact with water molecules with different mechanisms, at a molecular level.

Nevertheless these intrinsic and structural differences, the macroscopic behavior, as far as the dielectric point of view is concerned, of these heterogeneous systems are very similar, being the basis for a strict correlation between the dielectric response and the water molecules adsorbed, i.e., in other words, with the environmental humidity.

Humidity sensors based on resistive and/or capacitive response to water 
are based on this correlation and take advantage of the exceptionally large sensitivity reflected by changes of the electrical parameters in the presence of different amount of water.

\section{Synthesis of nanostructured polymers for gas sensors}

The size, shape, composition, hybrid, molecular engineering, assembly and nano-structures are the key parameters which characterize nanostructured polymers, drive their functions and allow to achieve applications in different fields.

The synthesis approaches are mainly based on a bottom-up procedure, starting from the monomers to arrive at the whole nanostructure by rationally tailoring experimental parameters, that readily and selectively lead to produce different types of micro/nano-materials with novel morphologies, with high performance in applications in different fields, such as electrochemical sensors, electro-chemiluminescent sensors, gene delivery agents.

Organic and organometallic polymers have been already investigated as sensing materials [8] and deeply investigated from a synthetic point of view $[9,10]$.

The most popular methods are wet chemistry and electrochemistry that are now discussed in a recent review [11] where the preparation of nanostructured materials, such as gold nanospheres, nanoplates and organic-inorganic hybrid colloid spheres, conducting polymer nanobelts and spherical colloids of coordination compounds is described on the basis of chemical reduction of the corresponding metal precursors $[12,13]$. A scheme of the synthesis routes for the achievement of nanoparticles of various origins [14] is shown in Fig. 1.

To bottom-up methods, belong the self-assembly procedure, electrochemical, template assisted, grafting, emulsion, gamma-radiation induced, and chemical oxidation.

The top-down methods, that involve electrospinning technology, LangmuirBlodgett deposition, osmosis and laser micro/nanopatterning are widely discussed and recently reviewed by some of us [15].

Some selected images of nano-polymers that could be used as sensitive polymeric materials for sensors are reported in Figs. 2 and 3.

However, this classification is sometimes ambiguous, because the two methods, i.e., bottom-up and top-down, are often complementary and the techniques to achieve nanoparticles frequently overlap [16]. Among the bottomup methods, the emulsion polymerization has been widely investigated for the achievement of polymeric nanoparticles for different applications. For example, polymethylmethacrylate and related polymeric nanospheres were obtained and assembled in photonic crystals domains $[17,18,19,20,21]$ and similar polymeric nanobeads were used for catalytic and bio -technological purposes [22, 23, 24]. A recent book comprehensively reviews the main features related to the development of conducting nanopolymers by a variety of synthetic methods and 


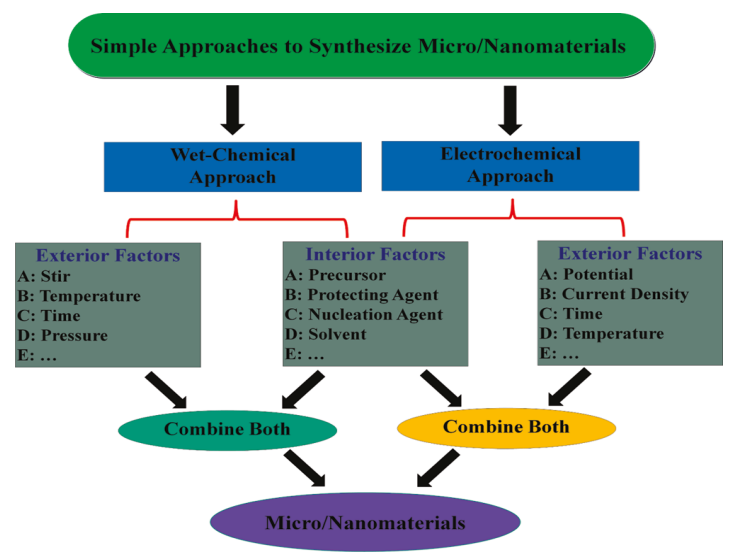

Figure 1: Schematic procedure for simple synthesis of nano- and microstructured polymers. Reproduced with permission from Guo and Wang, Acc. Chem, Res. 491-500 2011 Vol. 44.

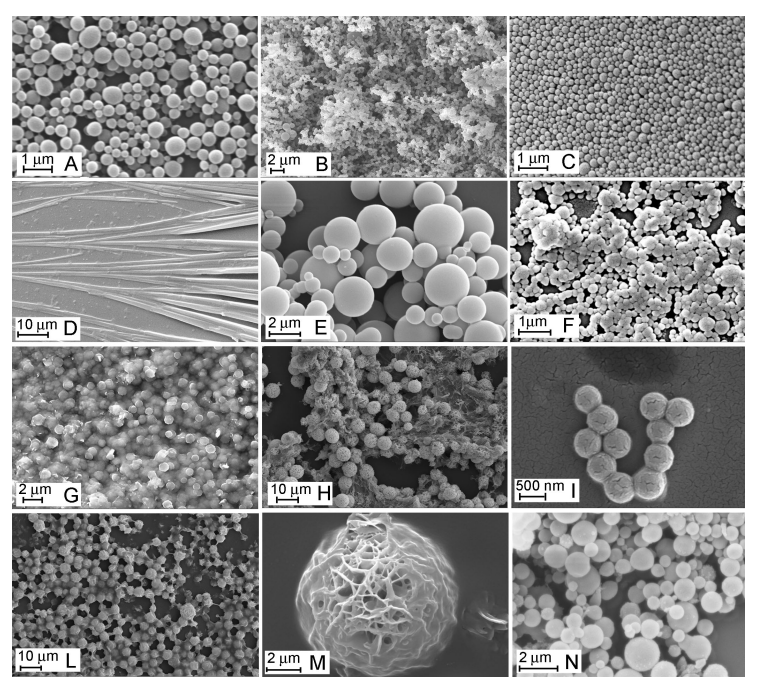

Figure 2: SEM images of different morphologies obtained for synthetic polymers (A): polymethylmethacrylate; (B): polystyrene; (C): polyphenylacetylene; (D) and (E): poly(N,N-dimethylpropargylamine derivatives; (F): Ptpolymetallayne) and biopolymers: (G): chitosan; (H), (I), (L) and (M): hyaluronic acid derivatives; $(\mathrm{N})$ : dextran. (Reprinted with permission from Chronopoulou L, Fratoddi I, Palocci C, Venditti I, Russo MV. Langmuir2009, copyright 2009 American Chemical Society). 


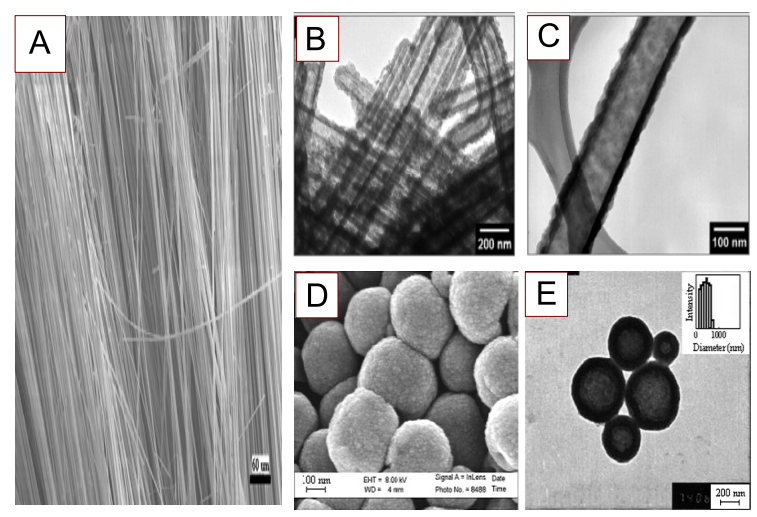

Figure 3: : Panel A: a large number of PANI arrays with average diameter of $1.2 \mu \mathrm{m}$ and highly ordered structure, produced by change of the aniline/acid concentration ratio. (Reprinted from Wu J, Tang Q, Li Q, Lin J, (2008) Polymer 49: 5262-5267, with permission from Elsevier). Panel B: TEM images of $\mathrm{PPy}$ nanotubes prepared in octane and enlargement $(\mathrm{C})$. (Adapted with permission from Jang J, Yoon H, (2005) Langmuir 21: 11484-11489 Copyright 2009 American Chemical Society). Panels D and E: SEM and TEM micrographs of PEDOT hollow aggregates (the exterior size distribution of hollow microsphere is shown in inset of panel E. (Reprinted from Xia Y, Wei M, Lu Y., (2009) Synth Met. 159: 372-376 with permission from Elsevier)

applications [25] and a recent review extensively reports about the development of nano-structured conducting polymers/nanocomposites, their characteristics and applications in sensors/biosensors and advances made in this field [26].

The actual main feature of conjugated polymers that make them suitable for chemical sensors is nanostructure. Nanostructured sensors systems are characterized by high sensitivity and selectivity, and find application in food quality control, pharmaceutical industries, medical diagnosis, environmental monitoring, and homeland security [25].

Nanosized and microsized particles of conjugated polymers have founded to have superior performance relative to conventional materials due to their much larger exposed surface area, reversible electrical properties due to redox behavior, mechanical flexibility and ease of preparation. Among others, the most investigated polymers are polyaniline [PANI] [27], polypyrrole [PPy] [28], polythiophene $[\mathrm{PTH}][29]$, polyparaphenylene [PPP], polyparaphenylenevinylene $[\mathrm{PPV}]$ and poly(3,4-ethylenedioxythiophene) $[\mathrm{PEDOT}]$ and polyphenylacetylene [PPA] [30].

The sensitivity of conducting polymer-based sensors is primarily determined by the difference in a specific property (generally, the capacitance or the electrical resistance) before and after exposure to a test target molecule (water 
molecules, for humidity sensors) and in this case the specific surface area of the conducting polymers plays a key role in determining the sensitivity of the sensors.

Composites of polymeric materials with metal nanostructures have also been investigated. Starting from preliminary studies [31, 32], a particular emphasis on their applications in sensor devices has been reported in a comprehensive review [33] where the trends in the synthesis and application of ICP (Intrinsically Conductive Polymers) composites in chemical sensing are highlighted. These authors demonstrate the versatility of these materials that can be utilized to develop sensing materials, with improved properties such as enhanced resistance to humidity, lower detection limits, increased sensitivity, lower sensing temperatures, and enhanced stability.

Another comprehensive review [34] highlights the fabrication of 1D multifunctionalized conducting polymer nanocomposites, where the components can be biological polymer materials, metal phthalocyanines and porphyrins and carbon nanotubes, metals, oxide nanomaterials, chalcogenides, etc. These composites find applications in a variety of electronic nanodevices and chemical and biological sensors.

In summary, nanotechnology provides the ability to work at the molecular level, atom by atom, to create structures with fundamentally new molecular organization.

In this context, nanostructured polymers have great potential for developing a new generation of chemical sensors to detect gas and vapor species and in particular water humidity. Nano-polymer-based sensors have the following properties: high sensitivity due to large surface to volume ratio, fast response, in the case of one-dimensional quantum wire nature, that makes its electronic properties very sensitive to gas adsorption, lower power consumption, which is ideal for persistent surveillance applications, small size and lightweight.

\section{Relative humidity [RH] sensors}

There is a wide number of contexts where it is important the control or the detection of humidity. They include environmental monitoring for meteorological services [35], agriculture for seed storage, industrial fields for chemical, biomedical [36], food industry [37] and electronics processing [38], as well as daily life for air conditioner in living rooms, hospitals.

Materials used for humidity sensing are extremely diversified, including organic polymers [39], ceramics [40], hybrid polymer/inorganic systems [41], composites [42], polymer electrolytes (polyelectrolytes) [41], each of them has its own merits and specific conditions of applications.

Humidity sensors utilize changes in physical properties (mainly in the electrical properties) of the sensitive element when exposed to different atmospheric humidity conditions of the surrounding environment, as a consequence of adsorption and/or desorption of water molecules. 
Based on measurement techniques, the most commonly used units for humidity measurements are Relative Humidity [RH], Dew/Frost point [D/F PT] and Parts Per Million [PPM].

Relative Humidity $[\mathrm{RH}]$ is defined as the amount of the actual water vapor density $e_{a}$ in the atmosphere as a percent of the amount of water vapor density $e_{s}$ the atmosphere could hold at that temperature and pressure, i.e.,

$$
R H=\frac{e_{a}}{e_{s}} \times 100 \%
$$

$\mathrm{RH}$ is a function of temperature, and thus it is a relative measurement.

Dew point is the temperature (above $0{ }^{\circ} \mathrm{C}$ ) at which the water vapor in a gas condenses to liquid water. Frost point is the temperature (below $0{ }^{\circ} \mathrm{C}$ ) at which the vapor condenses to ice. On the basis of these definitions, D/F PT is a function of the pressure of the gas, but is independent of temperature and is therefore defined as absolute humidity measurement.

Parts Per Million [PPM] represents water vapor content by volume fraction $[\mathrm{PPMv}]$ or, if multiplied by the ratio of the molecular weight of water to that of air, as weight ratio $[\mathrm{PPMw}]$. PPM is also an absolute measurement.

Absolute Humidity (vapor density) is defined as a ratio of the mass of water vapour in air to the volume of air expressed as:

$$
A B=\frac{m_{w}}{V}
$$

where $m_{w}$ is the mass of water vapor and $V$ is the volume of air.

Fig. 4 summarizes the most used units and shows the correlation among Relative Humidity [RH], Parts Per Million by volume [PPMv], and the Dew/Frost Point [D/F PT]. RH measurement covers higher humidity range, PPMv covers lower humidity range, and D/F PT covers all the humidity range. For daily life, Relative Humidity $[\mathrm{RH}]$ is the most frequently used unit because of its ease of understanding.

In this section, we will consider, from a general point of view, some examples of sensors based on differently organized nanostructured polymers, which are exploited, in the last few years, for the detection of relative humidity [RH].

Most of the sensing polymers are either hydrophilic or hydrophobic, which implies that they show considerable structural reorganization in presence of water. Hence, these materials exhibit, as a consequence of water adsorption, conductive or dielectric property changes with environmental humidity, which can be quantified for $\mathrm{RH}$ measurement.

Traditionally, polymeric humidity sensors are divided into two categories, i.e., resistive type and capacitive type [39]. However, the basic mechanism is essentially the same for both types, with the difference that, in the former, the physical parameter sensitive to the humidity variation is the electrical conductivity $\sigma$, while, in the latter case, is the electrical permittivity $\epsilon^{\prime}$. These parameters can be easily converted into resistance and capacitance respectively, taking into account the geometry of the sensor device. 


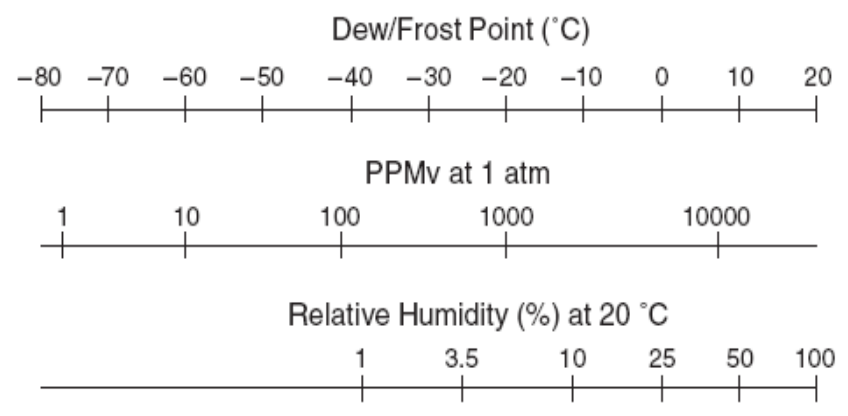

Figure 4: Correlation among humidity units: Relative Humidity (RH), Dew/Frost point (D/F PT), and Parts Per Million by volume fraction (PPMv). Reproduced with permission from Chen and Lu, Sensor Lett., 2005.

Basically, the nanosensor contains a pair of interdigitated electrodes covered by nanoporous polymer membranes. The resistance and/or capacitance between these electrodes vary at different humidity levels due to the water adsorption (mainly, capillary condensation) inside the nanopores.

As a matter of fact, in polymeric humidity sensors, the sensing element is essentially based on a porous polymer film filled with micro-pores and/or nano-pores differently interconnected for water vapor condensation.

Water condensation tends to take place within the porous structure of the polymer, the amount of condensed water being mostly dependent on the open pores volume, pore radius sizes and distributions. If, for sake of simplicity, we assume cylindrical pores, the pore radius at which capillary condensation begins to take place can be evaluated based on the Kelvin equation [43, 44], which states that this radius is given by

$$
r=\frac{2 \gamma M}{\rho R T \ln \frac{p_{s}}{p}}
$$

where $\gamma, M$ and $\rho$ are the surface tension $\left(72.75 \mathrm{dyn} / \mathrm{cm}\right.$ at $\left.20^{\circ} \mathrm{C}\right)$, molecular weight and density of the water, respectively. $p$ is the water vapor pressure and $p_{s}$ is the water vapor pressure at saturation. To a first approximation, the ratio $p / p_{s}$ equals the relative humidity $\mathrm{RH}$. $\mathrm{R}$ is defined as the gas constant and $\mathrm{T}$ is the absolute temperature. Water condensation occurs in all the pores with radius up to $r / 2$, at the given temperatures and constant pressures [45]. For a smaller $r$ value, or a lower temperature, water vapor condensation occurs in capillaries more easily (Fig. 5).

When there is adsorbed water vapor in the pore space, as a consequence of water condensation, its total volume fraction $\Phi$ will increase from 0 to 1 . Since water has very high permittivity at room temperature (about 80 ) compared to air (about 1), the effective dielectric constant of whole polymeric matrix will 


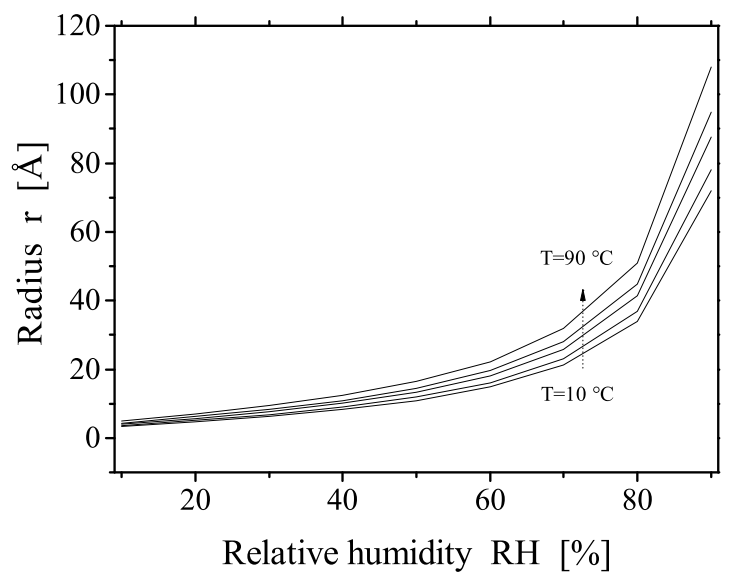

Figure 5: Pore radius of capillary condensation (Kelvin equation, eq.3).

increase accordingly. However, this means that, when RH decreases, desorption of water molecules from the liquid to the vapor phase requires extra energy, inducing, in the most cases of polymeric sensors, a large hysteresis and long recovery times. These processes are further complicated by the fact that water in highly confined systems behaves differently from bulk water and both water permittivity and water conductivity depend on the average size of the region where water is confined.

The above physical mechanisms can be recognized as a basic work principle of the various available humidity sensors.

In what follows, we will illustrate, on the basis of the effective medium approximation from a dielectric point of view, why a heterogeneous polymeric material, partially or fully embedded with water, represents an exceptionally suitable system with a dielectric, and conductometric too, response particularly sensitive to the amount of adsorbed water. This peculiar characteristic is enhanced by the nanostructured organization of the polymeric matrix that, because of its different geometric arrangement, allows to water to be adsorbed on an extraordinary large surface and, at the same time, inside nanopores and nanochannels where adsorbed water easily condensates in a bulk phase.

We will analyze these peculiar properties of water embedded nanostructured polymer systems on the basis of the dielectric response in different waterpolymer mixtures, differently organized by a geometric point of view.

However, in the present systems, it is worth noting that a rigorous dielectric description is made rather difficult by two circumstances, i.e., the high volume fraction of the aqueous phase and the fact that its geometrical distribution within the polymeric matrix is rather complex and, in principle, completely ignored. Therefore, we are forced to consider approximate theoretical framework 
which allows, at the same time, to treat this kind of systems with a reasonable degree of accuracy.

Among the different dielectric models proposed for dealing with these highly heterogeneous systems in the light of the effective medium theory approximation [46], we will consider different mixture equations, that work ignoring the detailed geometrical arrangement of the dispersed phase in the continuous matrix.

The first of these equations overcomes the requirement of low to moderate volume fraction but, at the same time, forces the dispersed phase to be spherical in shape with no interconnection between water regions.

In this case, the resulting complex dielectric constant $\epsilon^{*}(\omega)$ of the whole system, in the light of the effective medium theory approximation [46], composed by aqueous spheres of complex dielectric constant $\epsilon_{w}^{*}(\omega)=\epsilon_{w}+\sigma_{w} /\left(i \epsilon_{0} \omega\right)$ dispersed in a matrix of complex dielectric constant $\epsilon_{p}^{*}(\omega)=\epsilon_{p}$, at the volume fractions $\Phi$ and $(1-\Phi)$, respectively, is given by

$$
\frac{\epsilon^{*}(\omega)-\epsilon_{p}^{*}(\omega)}{\epsilon_{m}^{*}(\omega)-\epsilon_{p}^{*}(\omega)}\left(\frac{\epsilon_{m}^{*}(\omega)}{\epsilon^{*}(\omega)}\right)^{\frac{1}{3}}=1-\Phi
$$

The second mixture equation, which ignores the geometric details of the two phases, is suited for systems where the filler phase (water in this case) exhibits a high degree of interconnections through the polymeric matrix (interpenetrating network structure). Based on the so-called "aggregate topology", a mixture equation for a cubic-grid structure, including higher multipole moments has been proposed by Stolzle et al. [47] and takes the form [48]

$$
\epsilon^{*}(\omega)=\frac{1}{0.75}\left((1-\Phi) \epsilon_{e f f}^{*}\left(\Phi_{c}\right)+(\Phi-0.25) \epsilon_{p}^{*}(\omega)\right)
$$

where

$$
\epsilon_{e f f}^{*}\left(\Phi_{c}\right)=\left((1-\Phi)\left[\epsilon_{m}^{*}(\omega)\right]^{a}+\Phi\left[\epsilon_{p}^{*}(\omega)\right]^{a}\right)^{1 / a}
$$

with $\Phi_{c}=0.25$ and $a=1.6 \Phi+0.265$. In this case, we remark that both matrix and filler phases are interconnected.

In Fig. 6, we report the typical behavior of the permittivity $\epsilon^{\prime}$ and the electrical conductivity $\sigma$ as a function of the water volume fraction $\Phi$, for the two different polymeric structures described by eq. 4 and eqs. 5 and 6 , respectively. As can be seen, in both cases, the electrical response of the system is highly sensitive to the total water content.

The permittivity $\epsilon^{\prime}$ and the electrical conductivity $\sigma$ can be easily converted into the capacitance $C$ and the resistance $R$ by pure geometrical factors (the effective surface and the thickness of the polymeric layer of the sensitive element).

A more complex equation, originally developed to take into account the dielectric behavior of microemulsions close to the percolation threshold $[49,50$, 

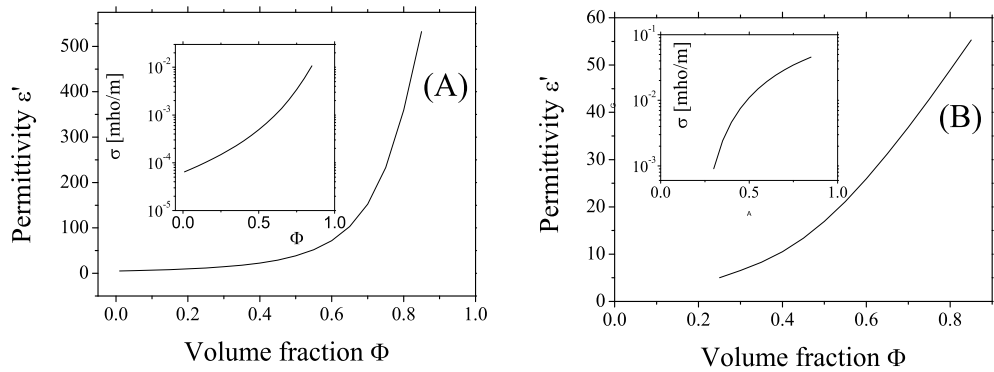

Figure 6: Panel (A): The permittivity $\epsilon^{\prime}$ of a porous polymeric matrix embedded of water as a function of the water volume fraction $\Phi$ at the frequency of $10 \mathrm{kHz}$, according to the Brugman-Hanai model (eq. 4). The phase parameters are: $\epsilon_{p}=75 ; \sigma_{p}=0.01 \mathrm{mho} / \mathrm{m} ; \epsilon_{m}=5 ; \sigma_{m}=0.00001 \mathrm{mho} / \mathrm{m}$. The inset shows the electrical conductivity $\sigma$ as a function of the volume fraction $\Phi$. Panel (B): Permittivity $\epsilon^{\prime}$ and electrical conductivity $\sigma$ calculated as in (A), according to the interpenetrating network structure model (eqs. 5 and 6 )

51], which explicitly introduces the porosity $P$ of the polymeric matrix, reads

$$
(1-P) \frac{\epsilon_{p}^{* 1 / t}-\epsilon^{* 1 / t}}{\epsilon_{p}^{* 1 / t}+\frac{\varphi_{p}}{1-\varphi_{p}} \epsilon^{* 1 / t}}+\varphi_{w} P \frac{\epsilon_{w}^{* 1 / t}-\epsilon^{* 1 / t}}{\epsilon_{p}^{* 1 / t}+\frac{\varphi_{p}}{1-\varphi_{p}} \epsilon^{* 1 / t}}+\left(1-\varphi_{w}\right) P \frac{\epsilon_{a}^{* 1 / t}-\epsilon^{1 / t}}{\epsilon_{a}^{* 1 / t}+\frac{\varphi_{p}}{1-\varphi_{p}} \epsilon^{* 1 / t}}=0
$$

where $\epsilon_{p}^{*}, \epsilon_{w}^{*}$ and $\epsilon_{a}^{*}$ are the complex dielectric constant of the polymer phase, water and air, respectively, $P$ is the porosity, $\varphi_{w}$ and $\varphi_{p}$ the volume fraction of adsorbed water and the percolation volume fraction, respectively and, finally, $t$ is the non-linearity correction factor.

In this framework, the porosity $P$ (the total volume of the pore space divided by the total sample volume) is defined as

$$
P=\int_{0}^{1} \phi f(\phi) d \phi
$$

where the local porosity distribution $f(\phi)$ is defined as the probability density of finding porosity between $\phi$ and $\phi+d \phi$ inside the sample.

In Fig. 7 , we show the typical increase of the permittivity $\epsilon^{\prime}$, or conversely of the capacitance $C$, and of the electrical conductivity $\sigma$, or conversely the resistance $R$, as a function of the amount of water adsorbed into the polymeric matrix, for different values of the porosity $P$. Also in this case, the overall system is extremely sensitive to the amount of adsorbed water, as can be seen, as an example, in Fig. 7, where both the permittivity and the electrical conductivity vary over an exceptionally wide range as a function of the total water content. 

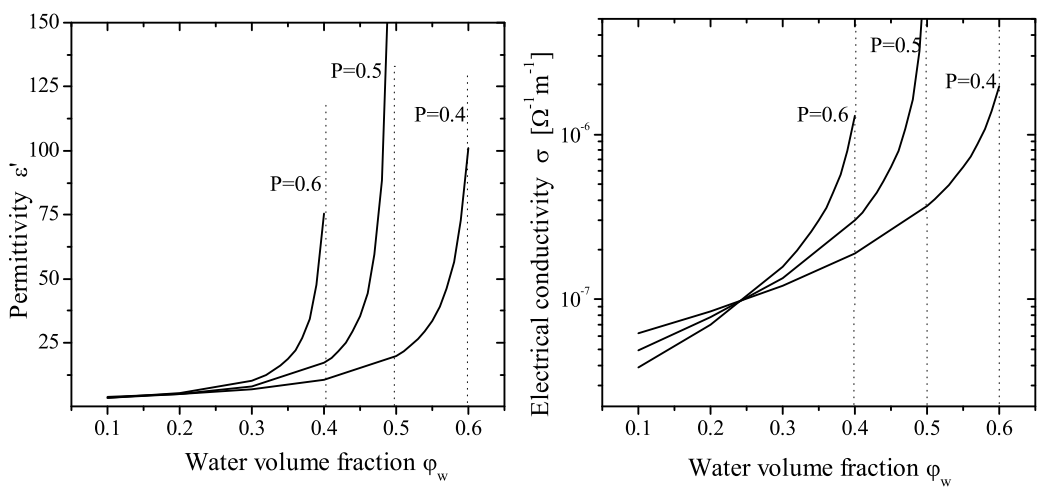

Figure 7: Left Panel: The permittivity $\epsilon^{\prime}$ of a porous polymeric matrix embedded of water as a function of the water volume fraction $\varphi_{w}$ at the frequency of $1 \mathrm{kHz}$, according to the percolative model (eq. 7). The phase parameters are: $\epsilon_{p}=5 ; \sigma_{p}=0.0000001 \mathrm{mho} / \mathrm{m} ; \epsilon_{w}=80 ; \sigma_{w}=0.01 \mathrm{mho} / \mathrm{m}$. Right panel: the electrical conductivity $\sigma$ as a function of the volume fraction $\varphi_{w}$. Calculations have been carried out for three different values of the porosity $P$

Moreover, sensors based on the electrical characterization of the sensing element offer the further advantage that the measuring frequency of the capacitance and/or the electrical conductivity can be properly tuned in order to obtain the most appropriate response, as far as the sensitivity of the measuring devise is concerned. As an example, in Fig. 8, we show the frequency dependence, in the range from $1 \mathrm{kHz}$ to $1 \mathrm{GHz}$, of the permittivity and electrical conductivity calculated on the basis of eq. 7. As can be seen, the whole response is markedly dependent on the frequency, even if, as pointed out by Das et al. [52], at very high frequencies, the measured capacitance is mainly determined by the parasitic capacitance. On the other hand, at lower frequencies, the measured capacitance truly represents the active capacitance for humidity sensing.

Among the different models proposed in the light of the dielectric theory for dealing with these highly heterogeneous systems [53], the simplest relationship which, to a large extent, ignores the details of the material composition and considers the sum of the dipolar factors associated with the different phases present in the system, each of them weighted by the respective volume fractions, has been proposed by Bruggeman [54, 55]. From a general point of view, for a two-phase system, characterized by two components of complex dielectric constant $\epsilon_{1}^{*}(\omega)$ and $\epsilon_{2}^{*}(\omega)$, at the volume fractions $\Phi_{1}$ and $\Phi_{2}=\left(1-\Phi_{1}\right)$, respectively, the resulting complex dielectric constant $\epsilon^{*}(\omega)$ of the whole system, 

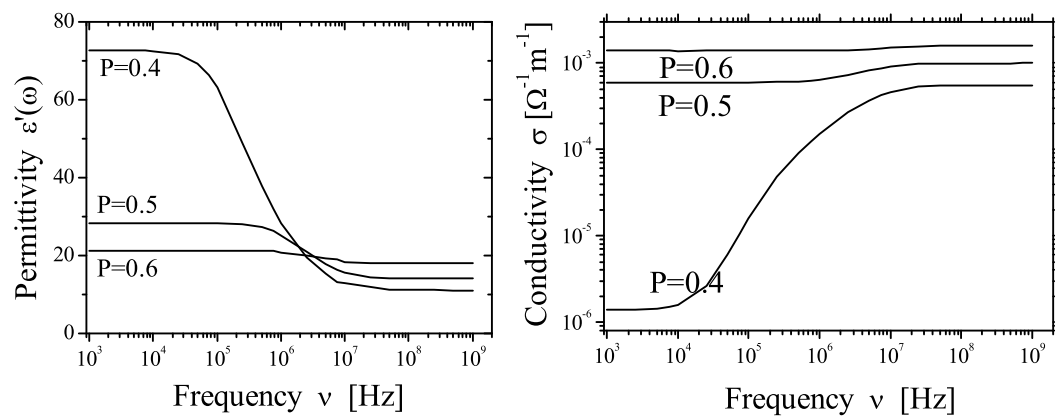

Figure 8: Left Panel: The permittivity $\epsilon^{\prime}$ of a porous polymeric matrix embedded of water as a function of frequency from $1 \mathrm{kHz}$ to $1 \mathrm{GHz}$ at water volume fraction $\varphi_{w}=0.59$, according to the percolative model (eq. 7 ). The phase parameters are: $\epsilon_{p}=5 ; \sigma_{p}=0.0000001 \mathrm{mho} / \mathrm{m} ; \epsilon_{w}=80 ; \sigma_{w}=0.01 \mathrm{mho} / \mathrm{m}$. Right panel: the electrical conductivity $\sigma$ as a function of frequency. Calculations have been carried out for three different values of the porosity $P$.

in the light of the effective medium theory approximation, is given by

$$
\Phi_{1} \frac{\epsilon_{1}^{*}(\omega)-\epsilon^{*}(\omega)}{\epsilon_{1}^{*}(\omega)+2 \epsilon^{*}(\omega)}+\Phi_{2} \frac{\epsilon_{2}^{*}(\omega)-\epsilon^{*}(\omega)}{\epsilon_{2}^{*}(\omega)+2 \epsilon^{*}(\omega)}=0
$$

This equation, ignoring the geometric details of the two phases, lets itself to be generalized to a highly porous and interconnected system. According to Haslund et al. [56] and Hilfer [57], on the basis of the local porosity theory [58, $59,60]$, which includes additional structural information through the geometric functions $\mu(\phi)$ and $\lambda(\phi)$, eq. 9 can be written as

$$
\begin{array}{r}
\int_{0}^{1} \frac{\epsilon_{w e}^{*}(\omega)-\epsilon^{*}(\omega)}{\epsilon_{w e}^{*}(\omega)+2 \epsilon^{*}(\omega)} \lambda(\phi) \mu(\phi) d \phi+ \\
\int_{0}^{1} \frac{\epsilon_{p e}^{*}(\omega)-\epsilon^{*}(\omega)}{\epsilon_{p e}^{*}(\omega)+2 \epsilon^{*}(\omega)}[1-\lambda(\phi)] \mu(\phi) d \phi=0
\end{array}
$$

where $\epsilon_{w e}^{*}(\omega)$ and $\epsilon_{p e}^{*}(\omega)$ are the effective complex dielectric constant of the aqueous (conducting) phase and the polymeric (non-conducting) network with a local porosity $\phi$. Eq. 10 has been proposed as a generalization of effective medium theories [61], based on an extension of geometric characterization by using local geometry distribution [57,62], whose validity fails in either the pore scale length tends to zero (pores become strongly correlated) or to infinity (the medium is globally connected). However, eq. 10 holds over an intermediate range of pore size.

In this case, as we have above stated, the system is characterized by two 
geometrical functions, $\mu(\phi)$ and $\lambda(\phi)$ which take into account the local porosity distribution and the local percolation distribution in the whole system.

Both the dielectric constants $\epsilon_{p e}^{*}(\omega)$ and $\epsilon_{w e}^{*}(\omega)$ depend on the bulk complex dielectric constants of the two components, $\epsilon_{w}^{*}$ for the water phase and $\epsilon_{p}^{*}$ for the polymeric phase, according to the relationships [56, 58]

$$
\begin{gathered}
\epsilon_{w e}^{*}(\omega)=\epsilon_{w}^{*}(\omega)\left(1-\frac{1-\phi}{\left[1-\epsilon_{p}^{*}(\omega) / \epsilon_{w}^{*}(\omega)\right]^{-1}-\frac{1}{3} \phi}\right) \\
\epsilon_{p e}^{*}(\omega)=\epsilon_{p}^{*}(\omega)\left(1-\frac{\phi}{\left[1-\epsilon_{w}^{*}(\omega) / \epsilon_{p}^{*}(\omega)\right]^{-1}-\frac{1}{3}(1-\phi)}\right)
\end{gathered}
$$

Here, $\epsilon_{p}^{*}(\omega)$ and $\epsilon_{w}^{*}(\omega)$ are the complex dielectric constants of the polymeric matrix and of the aqueous phase, respectively.

Because of the range of frequencies investigated and considering that the porous matrix has negligible electrical conductivity $\left(\sigma_{p} \simeq 0\right)$ and its permittivity $\epsilon_{p}$ is practically independent of frequency and temperature, the following approximations

$$
\begin{gathered}
\epsilon_{p}^{*}(\omega)=\epsilon_{p} \\
\epsilon_{w}^{*}(\omega)=\epsilon_{w}+\frac{\sigma_{w}}{i \omega \epsilon_{0}}
\end{gathered}
$$

hold.

Eq. 10 contains the two functions that define the porous structure of the system. They are the local porosity distribution $\mu(\phi)$ and the local percolation probability $\lambda(\phi)$. As far as the function $\lambda(\phi)$ is concerned, we chose a dependence given by

$$
\lambda(\phi)=\phi^{\gamma}
$$

with the limiting values $\lambda(\phi=0)=0$ and $\lambda(\phi=1)=1$. For sake of simplicity, for the function $\mu(\phi)$, we assume a Gaussian dependence with expected value $\Phi$ and variance $s^{2}$.

Consequently, the whole dielectric behavior of the polymeric system is characterized by three parameters, $\Phi, \gamma$ and $s^{2}$, all of them depending on the geometry of the structural organization of the polymeric matrix.

In Fig. 9, we show the typical behavior of the permittivity $\epsilon^{\prime}$ and the electrical conductivity $\sigma$ as a function of the frequency, for different values of the parameter $\gamma$. Fig. 10 shows the increment of the permittivity and the conductivity as a function of the total fraction of water present in the system, i.e., proportional to the relative humidity.

A final comment to conclude this section is in order. We have considered different dielectric models proposed in literature to justify the dielectric behavior of highly heterogeneous systems. Whatsoever arranged the structure of the polymeric material is, we show that the dielectric response of the system is highly sensitive to the presence of water embedding the porous structured polymer material, justifying its use as sensitive element in humidity sensor devices. 

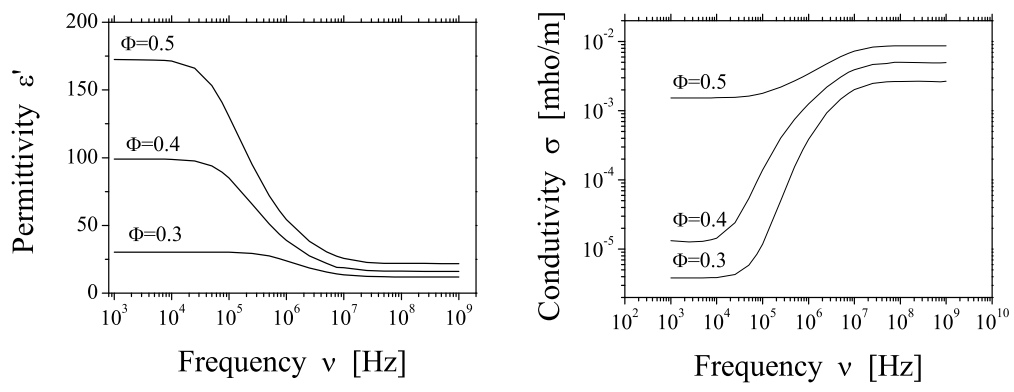

Figure 9: Left Panel: The permittivity $\epsilon^{\prime}$ of a porous polymeric matrix embedded of water as a function of frequency from $1 \mathrm{kHz}$ to $1 \mathrm{GHz}$ at water volume fraction for different values of the parameter $P$, according to the percolation model (eq. 10). The phase parameters are: $\epsilon_{p}=5 ; \sigma_{p}=0.0000001$ $\mathrm{mho} / \mathrm{m} ; \epsilon_{w}=80 ; \sigma_{w}=0.01 \mathrm{mho} / \mathrm{m}$. Right panel: the electrical conductivity $\sigma$ as a function of frequency.

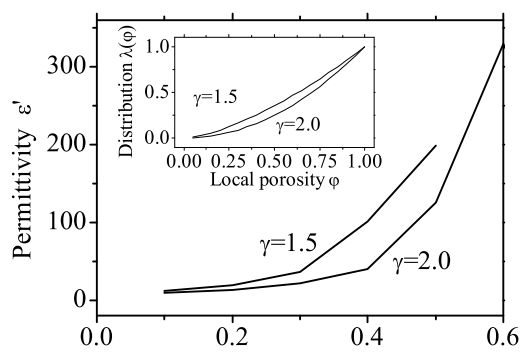

Total Fraction of adsorbed water $\Phi$

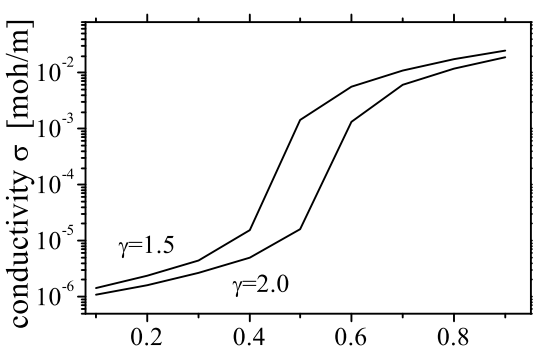

Total Fraction of adsorbed water $\Phi$

Figure 10: Left Panel: The permittivity $\epsilon^{\prime}$ of a porous polymeric matrix embedded of water as a function of total water volume fraction $\Phi$, at the frequency of $1 \mathrm{kHz}$, according to the percolative model (eq. 10) for two different values of the exponent $\gamma$. The phase parameters are: $\epsilon_{p}=5 ; \sigma_{p}=0.0000001 \mathrm{mho} / \mathrm{m}$; $\epsilon_{w}=80 ; \sigma_{w}=0.01 \mathrm{mho} / \mathrm{m}$. The inset shows the percolation distribution function $\lambda(\phi)$ as a function of the local porosity $\phi$. Right panel: the electrical conductivity $\sigma$ as a function of total water volume fraction $\Phi$. 


\section{Characteristics and properties of $\mathrm{RH}$ nanos- tructured polymer-based sensors}

Some examples of RH nanostructured polymer sensors, selected from the wide literature on this issue, based on different polymeric materials and different device transducers are hereafter reviewed, with the aim of representing the efforts made in this area of research, being, however, aware that the review will not be exhaustive, due to the amplitude of research in this field.

These examples are chosen with the aim of offering a number of selected cases to illustrate the wide variety of polymeric materials that obey to this phenomenology.

Works on humidity sensing mechanism of most widely used impedance type sensors have been carried out using dielectric impedance spectroscopy [63, 64, $65,66,67,68]$.

A good humidity sensor property has been shown by nanostructured hybrid films synthesized from hydroxyl propyl methyl cellulose [HPMC] and tetra ethoxy ortho silicate [TEOS] obtained by microwave assistance method [69]. The formation of HPMC/TEOS amorphous nano-hybrid film (film thickness between 20 and $24 \mu \mathrm{m}$ with a surface roughness od $120 \mathrm{~nm}$ ) resulted in a material with a good linear sensor response, a low hysteresis $(<1 \%)$, a fast response $(5 \mathrm{~s})$ and a good recovery time $(10 \mathrm{~s})$.

Lin et al. [70] investigated the relationship between dynamic water adsorption and impedance response of an electrospun polyaniline [PANI] nanofiber humidity sensor by quartz crystal microbalance $[\mathrm{QCM}]$ measurements. Nyquist plots and Bode plots of the nanofiber sensors were obtained at different humidity levels, and explained by taking into account the morphology of the nanofibers. An electrical equivalent circuit was developed and used to explore the sensing mechanism of the nanofibers sensor, whose effectiveness was verified by good agreement between measured and calculated results.

The QCM exploits the properties of piezoelectric materials to modify their frequency fluctuation according to variation of the viscoelastic coefficient of the membrane deposited on their electrodes. The frequency variation is given by $[71]$

$$
\Delta \nu=-\nu_{q}^{3 / 2}\left(\frac{\rho_{l} \eta_{l}}{\pi \rho_{q} \mu_{q}}\right)^{1 / 2}
$$

where $\rho_{l}$ and $\eta_{l}$ are the membrane density and viscosity, respectively, $\rho_{q}$ and $\mu_{q}$ are the quartz density and the shear modulus, respectively, with $\nu_{q}$ its fundamental frequency. To a first approximation, when the adhesion of the membrane on the quartz is particularly tenacious, the frequency change is induced by the increased mass of the sensitive membrane due to the captured moisture and is given by

$$
\Delta \nu=-2 \nu_{q}^{2} \frac{1}{\sqrt{\rho_{q} \mu_{q}}} \frac{\Delta M}{A}
$$




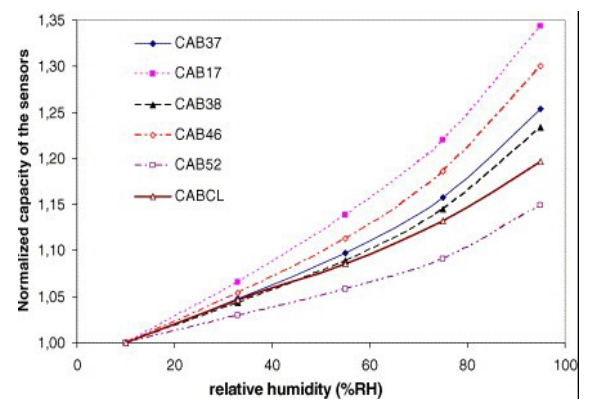

Figure 11: Normalized capacity of the sensors as a function of the relative humidity, for different type of cellulose acetate butyrate. The capacity of each sensors is measured with a LCR meter at a frequency of $10 \mathrm{kHz}$ and a voltage of $1 \mathrm{~V}$. Humidity range from 10 to $95 \% \mathrm{RH}$ at $25^{\circ} \mathrm{C}$ is generated with a SECASI climate chamber. Polymers have different molecular weight and different acetyl/butyril percentage ratio [A/B ratio]; $\mathrm{CAB} 52=16 \mathrm{kD}, \mathrm{A} / \mathrm{B}=3 / 52$; $\mathrm{CAB} 38=20 \mathrm{kD}, \mathrm{A} / \mathrm{B}=13 / 35 ; \mathrm{CAB} 37=70 \mathrm{kD}, \mathrm{A} / \mathrm{B}=14 / 32 ; \mathrm{CAB} 46=20 \mathrm{kD}$, $\mathrm{A} / \mathrm{B}=2 / 46 ; \mathrm{CAB} 17=20 \mathrm{kD}, \mathrm{A} / \mathrm{B}=30 / 17$. CABCL is a formulation of $50 \%$ of CAB52 mixed with $25 \%$ of CAB17 and $25 \%$ of CAB37.

where $\Delta M$ is the mass variation adsorbed on the surface of the membrane and $A$ its active area.

In the case of polymeric materials, the QCM does not only take advantage of the chemical intrinsic properties of the active membrane, but also of the increased specific surface area and of its porosity. The behavior of nanobeads of polyphenylacetylene and poly (phenylacetylene -(2- hydroxyethyl methacrylate -Co)) membranes as sensitive layer exposed to different values of RH has been discussed in Ref. [72].

Jaruwongrungsee et al. [73] proposed a layer of PANI micro- and nanodots as sensitive layer on quartz microbalance. The polymeric material was, in this case, deposited by means of an electrospinning technique which allows to reduce both the response and the recovery time.

Polymers such as polyimide [74, 75, 76, 77] and polymethyl metacrylate [PMMA] [78, 79, 80], polyethylene terephthalate [PET] [9] and polysulfone [PSF] $[81,82]$ are some of the materials employed in capacitive humidity sensors. A sensor based on cellulose acetate butyrate [CAB] having good characteristics (high sensitivity, small hysteresis, short response time and long term stability, with moreover good chemical resistance) has been realized by Ducere et al. [83]. Fig. 11 shows the capacitive response of different types of [CAB] is shown as a function of the relative humidity, for different content of butyryl (from 17 to $52 \%$ ) and different molecular weight (from 20 to $40 \mathrm{kD}$ ). This sensor works effectively from 0 to $100 \% \mathrm{RH}$ and from 40 to $120{ }^{\circ} \mathrm{C}$. Furthermore it is robust enough to be used in many applications such as industrial processes. 


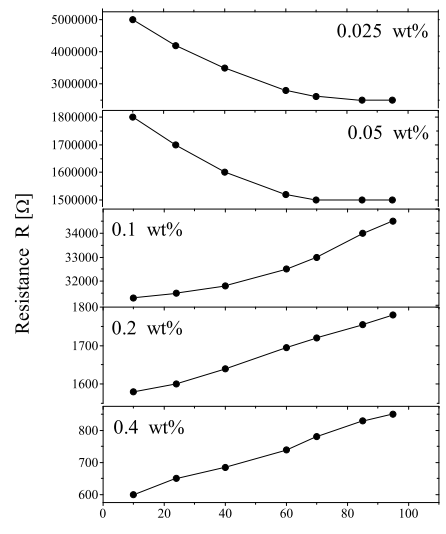

Relative humidity RH [\%]

Figure 12: A cumulative plot of the resistance $R$ as a function of the relative humidity $R H$ for different values of the carbon nanotube concentration, from 0.025 to $0.4 \mathrm{wt} \%$. Data taken at room temperature at the frequency of $20 \mathrm{kHz}$ with $1 \mathrm{~V}_{\text {rms }}$ test signal. Redrawn and adapted from Ref. [84]

To enhance the sensitivity of polymide-based resistive sensors, Yoo et al. [84] prepared a nanocompsite film where plasma-treated multiwall carbon nanotubes [CNT] were blended with polymide [p-MWCNT/PI film]. The electrical response of the film depends on the carbon nanotube content. This dependence is rather complex, showing a $\triangle R / \triangle R H<0$ for small $\mathrm{CNT}$ concentration and $\triangle R / \triangle R H>0$ for large CNT concentration.

When the concentration is higher than a critical value (around $0.1 \mathrm{wt} \%$ ), the sensor exhibits a very good linearity over a wide humidity range. In this case, the sensitivity $S$ is defined as

$$
S=\frac{\triangle R / R_{0}}{\triangle(R H \%)}
$$

where $\triangle R=R_{R H}-R_{0}$ with $R_{R H}$ and $R_{0}$ the steady-state resistance when the device is exposed to different $R H$ values and its initial resistance value. Typical values range from $0.00127 R H^{-1}$ at a carbon nanotube concentration of 0.1 wt\% to $0.00466 R H \%^{-1}$ at a nanotube concentration of $0.4 \mathrm{wt} \%$. A cumulative plot of the resistance $R$ as a function of the relative humidity is shown in Fig. 12 .

Polyaniline [PANI] nanofiber humidity sensors were prepared by Lin et al. [85] through electrospinning [ES] from the N,N-dimethylformamide solution of PANI, poly(vinyl butyral) [PVB] and poly(ethylene oxide) [PEO]. Humidity response of the nanofiber sensors was investigated by measuring their impedance at different humidity levels at room temperature and the main results are shown 


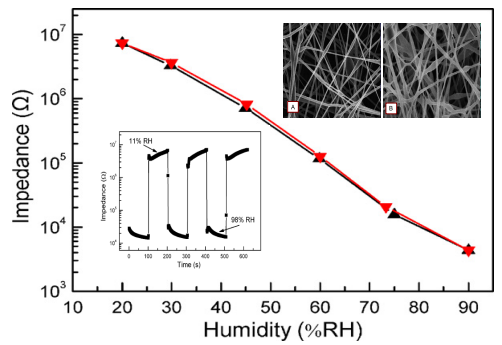

Figure 13: Humidity responses of the PANI nanofiber humidity sensor during the humidification (triangleup) and desiccation (triangledown) processes (ES solution: [PANI-PSSA] $=20 \mathrm{mg} / \mathrm{mL}$, PANI-PSSA $/ \mathrm{PEO} / \mathrm{PVB}=20 / 3 / 14$ (mass ratio), heated at $100{ }^{\circ} \mathrm{C}$ for $2 \mathrm{~h}$ ). Bottom inset: Response transients of PANI nanofiber humidity sensor (ES solution: [PANI- PSSA] $=20 \mathrm{mg} / \mathrm{mL}$, PANI-PSSA $/ \mathrm{PEO} / \mathrm{PVB}=20 / 3 / 14$ (mass ratio), heated at $100{ }^{\circ} \mathrm{C}$ for $2 \mathrm{~h}$ ). Upper inset: SEM images of nanofibers prepared from ES solutions of (a) $[$ PANI- PSSA $]=20 \mathrm{mg} / \mathrm{mL},[\mathrm{PVB}]=30 \mathrm{mg} / \mathrm{mL}$ and (b) [PANI-PSSA $]=20$ $\mathrm{mg} / \mathrm{mL},[\mathrm{PVB}]=30 \mathrm{mg} / \mathrm{mL}$. All the nanofibers were heated at $100{ }^{\circ} \mathrm{C}$ for 2 h. Redrawn and adapted from Ref. [85]

in Fig. 13. Sensors exhibited a small hysteresis of only 1\% RH under middle humidity, and good sensing linearity on a semi-logarithmic scale.

A similar approach has been carried out by Li et al. [86], who obtained by electrospinning and heat treatment nanofibers (with a diameter of 250-500 $\mathrm{nm}$ ) of a composite of a silicon-containing polyelectrolyte polyethylene oxide and polyaniline [PANI]. Nanofibers showed impedance change from about 6 $\mathrm{M} \Omega$ to $25 \mathrm{k} \Omega$ with the increment of the relative humidity from 22 to $97 \%$ at room temperature. Other interesting characteristics are fast reversible response with a small hysteresis (about $2 \% \mathrm{RH}$ ) and, moreover, a short response time (between $7 \mathrm{~s}$ and $19 \mathrm{~s}$ for adsorption and desorption in the range 33 to $97 \%$ $\mathrm{RH})$.

Among polymeric materials, polymer electrolytes (polyelectrolytes) have been the most widely employed as resistive-type humidity sensors, owing to their ion-conducting characteristics $[87,88]$. They respond to humidity variations by increasing their ionic mobility or changing the charge carrier concentration $[89,90]$.

A number of studies have led to humidity sensors based on polyelectrolytes [PEs] that, in some cases, are very sensitive in a wide range of relative humidity and very stable at high concentrations of water vapor [78, 91, 92, 93, 94].

However, being generally hydrophilic, polyelectrolytes tend to be soluble in water, resulting in a large hysteresis and, moreover, giving rise to a weak perdurability of the sensor device as a whole. This inconvenience can be easily overcome by modifying polyelectrolytes to make them insoluble in water [78, 95]. Amine-containing polyelectrolytes were prepared by Lee et al. [96] with 


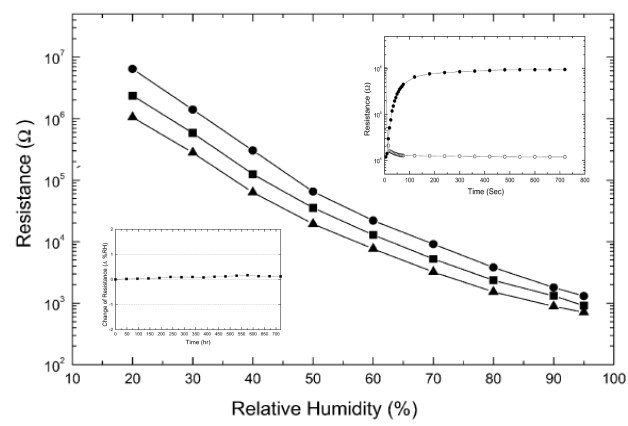

Figure 14: The resistance dependence on relative humidity of humidity sensor obtained from VTBPC/METAC/2-EHA/VP $=3 / 2 / 2 / 3$ at $(\bullet) 15{ }^{\circ} \mathrm{C}$; $25{ }^{\circ} \mathrm{C}$; and $(\boldsymbol{\Lambda}) 35{ }^{\circ} \mathrm{C}$ at $1 \mathrm{kHz}$ and $1 \mathrm{~V}$. Upper inset: Response time of the humidity sensor obtained from VTBPC/METAC/2-EHA/VP $=2 / 2 / 2 / 4 ;(\bullet)$ absorption and $(\bigcirc)$ desorption process at $25{ }^{\circ} \mathrm{C}$. Bottom inset: Long-term stability of the sensor under $90 \% \mathrm{RH}$ at $80{ }^{\circ} \mathrm{C}$ measured at $70 \% \mathrm{RH}$ of humidity sensor obtained from the cross-linked copolymer VTBPC/METAC/2-EHA/VP $=3 / 2 / 2 / 3$. Redrawn and adapted from Ref. [96]

different contents of vinylbenzyltributylphosphonium chloride [VTBPC], (2(methacryloyloxy)ethyl)trimethyl ammonium chloride [METAC], 2-ethylhexylacrylate [2-EHA] and 4-vinylpyridine [4-VP]. Resistance versus relative humidity characteristics of the sensor were measured for an adsorption process, at $20 \% \mathrm{RH} 95 \% \mathrm{RH}$, and for a desorption process, at $95 \% \mathrm{RH} 20 \% \mathrm{RH}$ at $1 \mathrm{~V}$, $1 \mathrm{kHz}$ and $25^{\circ} \mathrm{C}$. The temperature dependence was measured at temperatures of 15,25 and $35^{\circ} \mathrm{C}$ at $1 \mathrm{~V}$ and $1 \mathrm{kHz}$. Typical results are shown in Fig. 14. Temperature coefficient between 15 and $35^{\circ} \mathrm{C}$ is 0.35 to $0.56 \% \mathrm{RH}$ per degree centigrade and the response time is $125-175$ s between $33 \% \mathrm{RH}$ and $94 \% \mathrm{RH}$.

The reduction of water solubility can be obtained with the application of hydrophobic groups by grafting or by the co-polymerization in order to apply a protective film $[95,78,91]$ or by cross-linking reaction $[97,92,98]$. Copolymerization of hydrophilic or hydrophobic monomers with other hydrophobic monomers and the influence of different parameters on the performance of cross-linked quaternized polyelectrolyte films have been studied by Wu et al. [99] and by Casalbore-Miceli et al. [100].

Nanoporous polymers based on 1,4-divinylbenzene [DVB] and methacrylato ethyl trimethyl ammonium chloride [DMC] were recently synthesized by free radical polymerization with a solvothermal route by Fei et al. [101]. Hydrophobic $[\mathrm{DVB}]$ and hydrophilic $[\mathrm{DMC}]$ monomers were used for constructing polymer electrolytes with different molar ratio. Fig. 15 collects the main results for PDVB/DMC with a molar ratio of DVB to DMC of 3:3, where the 


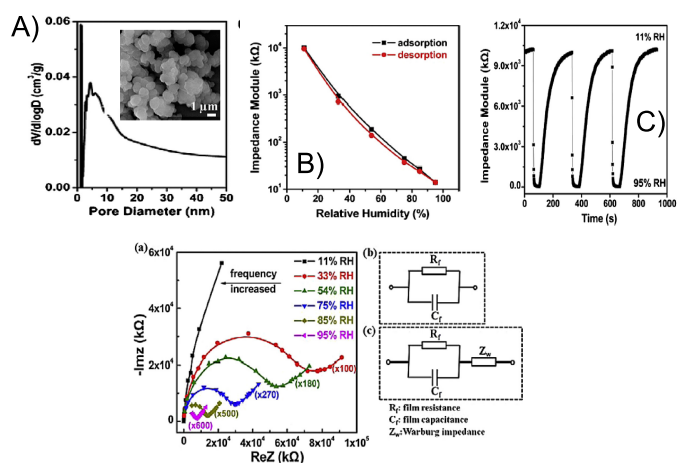

Figure 15: Panel A: pore diameter distribution; Panel B: humidity hysteresis curve; Panel C: response and recovery time; Bottom panel, left: complex impedance under different RH conditions; Bottom panel, right: equivalent circuit for complex impedance.

pore diameter distribution (panel A), the humidity hysteresis curve (panel B), the response and recovery curve (panel $\mathrm{C}$ ) and the complex impedances under different $\mathrm{RH}$ conditions (bottom panel a) together with the equivalent circuit for the complex impedance (bottom panel b) are shown.

Authors conclude that this cross-linked structure offers good stability of the polymer even at hight humidity level, showing changes of more than three orders of magnitude in the impedance response, over the whole humidity range, with little humidity hysteresis and rapid response.

The same authors [102] have realized a humidity switching sensor based on different hydrophobic polymers (poly(N-vinyl-2-pyrrolidone) [PVP], poly(vinyl alcohol) [PVA], hydroxyethyl cellulose [HEC]) that present different hydrophobic properties, resulting in different interactions with water.

Impedance of the sensors based on PVP, PVA and HEC shows a non linear response to relative humidity with switching humidity points at about 75,65 and $55 \% \mathrm{RH}$, respectively. Moreover, a mixture of these polymers may be used for controlling the switching properties. For example, sensors based on $\mathrm{PVP} / \mathrm{PVA}$ composite film shift their switching point from 75 to $60 \% \mathrm{RH}$ as the composition varied from PVP to PVP/PVA 1:1 molar ratio.

Recently, Kim et al. [103] synthesized partially sulfonated poly(styreneblock- methylbutylene) block copolymers [PSS-b-PMB] where the PSS chains provide hygroscopic nature. In this composite, the degree of water sorption can be easily tuned by adjusting sulfonation levels of the PSS chains. In this way, it is possible to accurately control sensor performance. In these sensors, changes in electrochemical properties upon exposure to environmental humidity can be attributed to the existence of water molecules in pools of $\mathrm{SO}_{3} \mathrm{H}$ groups which lead to proton transports along neighboring protic moieties, resulting 


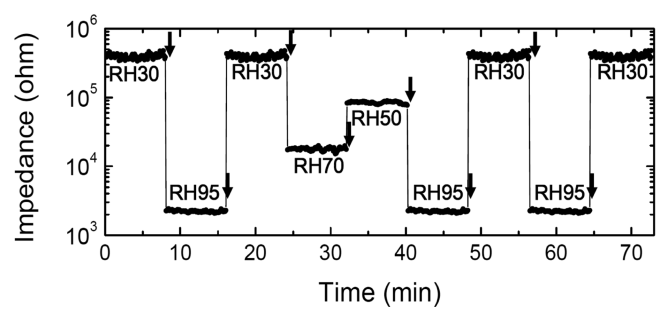

Figure 16: Impedance changes of a PSS-b-PMB film, monitored with repeated stepwise changes in RHs. For all measurements, an AC voltage of $20 \mathrm{mV}$ at a fixed frequency of $1 \mathrm{kHz}$ was supplied. The injection of air with different RHs was indicated in the figure with inverted arrows. Redrawn and adapted from Ref. [103]

in a decrease in the electrical impedance of the films. Typical changes in the impedance of PSS-b-PMB sensors are shown in Fig. 16, where the sensor response is monitored at $25^{\circ} \mathrm{C}$ as a function of time, upon switching $\mathrm{RH}$ from 20 to $95 \%$.

By means of a simple and easy procedure, a resistive-type cross-linked polyelectrolyte humidity sensitive material was realized by Lv et al. [104], who used 2-(dimethyl amino) ethyl metacrylate quaternized with n-butyl bromide and then copolymerized with 1.4-divinyl benzene. The resulting material exhibited a high sensitivity (impedance change from $10 \mathrm{M} \Omega$ to $1 \mathrm{k} \Omega$ in the humidity range 22 to $97 \% \mathrm{RH}$ ), small hysteresis (about $1 \% \mathrm{RH}$ ) and fast response (about 9 and $36 \mathrm{~s}$ for adsorption and desorption between $33 \%$ and $99 \% \mathrm{RH}$ ).

Recently, Pattananuwat et al. [105] produced a novel humidity sensor based on poly(pyrrole-co-formil pyrrole) $[\mathrm{P}(\mathrm{Py}$-co-Py) $]$ film prepared via spin coating during chemical polymerization (typical thickness of the film was of the order of $2 \mu \mathrm{m}$ with a surface roughness of about $1 \mathrm{~nm}$ and a conductivity of 0.02 $\mathrm{mho} / \mathrm{cm})$. The response of the film was highly sensitive to the RH change, the impedance changed from about $100 \mathrm{k} \Omega$ to $10 \Omega$ for $\mathrm{RH}$ varied from 15 to $95 \%$.

Moreover, the $\mathrm{P}(\mathrm{Py}-\mathrm{co}-\mathrm{Py})$ film responded rapidly, with a small hysteresis, when RH changed from 30 to $90 \%$ within $5 \mathrm{~s}$. These films showed a frequencydependent capacitance response with a rapid increase with increasing \% $\mathrm{RH}$. A detailed analysis of the dielectric behavior of poly pyrrole polymer has been carried out by Zhang et al. [65], showing that, in this case, electrical conduction is partially due to a dipolar contribution.

Besides, conducting polymers like poly(3,4-ethylenedioxythiophene) [PEDOT] or poly(3,4-ethylenedioxythiophenepoly(styrene-sulfonate) [PEDOT-PSS] exhibit higher moisture sensitivity, and significant reversible changes of impedance when exposed to humid conditions [106]. A resistive-type miniature humidity sensor made of an organic conjugated polymer based on PEDOT has been 
developed and fabricated using standard MEMS technology integrated with a polymerisation process. The electrical test characteristics showed that the sensor is sensitive to a RH range of $20 \%$ to $99 \%$ [107]. Further, Miyoshi et al. reported a flexible design configuration for a resistive humidity sensor based on a sandwiched porous hydrophobic poly-tetrafluoroethylene membrane structure deposited by a soft-MEMS technique applicable to physiological humidity ranges [108].

The capacitive-type sensor is superior in linearity of sensor output and in stability at high humidity to the resistive-type sensor. However, many problems, i.e., hysteresis, stability at high temperature and in a highly humid atmosphere and durability to some kinds of organic vapors, etc., still have to clarified. These aspects require a detailed knowledge of the mechanisms, at a molecular level, that control interactions of water in highly confined polymeric regions.

Capacitive sensors are based on hydrophobic polymer films that adsorb water by hygroscopicity [109]. Good candidates to this class of materials are cellulose acetate butyrate [CAB] [110], poly(methyl metacrylate) [PMMA] [111], poly(vinyl crotonate) [PVC] [112] and poly(ethylene terephthalate) [PETT] [113].

Their properties are recently reviewed by Chen and Lu [109], who discuss application of cross-linking in order to enhance the sensitivity of the film.

\section{Conclusions}

Nanostructured polymers represent a suitable class of materials to be employed as sensitive element in capacitive-type and conductive-type humidity sensors. This property is due to the fact that these systems, when embedded partially or totally of water caused by environmental humidity, undergo an exceptionally wide response in terms of changes in the electrical resistance and electrical capacitance, justifying their use as sensitive elements in humidity sensors.

We have analyzed, on the basis of the most appropriate dielectric models, the theoretical reasons by which this phenomenology occurs, considering various possible spatial arrangements assumed by nanostructured polymers and, for each of them, we have calculated, in the framework of the effective medium theory approximation, the permittivity and the conductivity as a function of the frequency and, moreover, as a function of the total amount of water embedded the system.

It worth noting that this analysis has been carried out for different porosity of the polymeric material, introducing two functions, the pore distribution and the local percolation distribution, that allow a detailed quantitative description of these highly heterogeneous systems.

Our results, for all the systems investigated, confirm that permittivity and electrical conductivity are electrical parameters to be successfully employed in the measurement of relative humidity. This conclusion is further supported by a brief review of recent humidity sensors based on nanostructured polymers. 


\section{References}

[1] J. Zhang, H. Sun, and P. X. Ma. Host-guest interaction mediated polymeric assemblies: Multifunctional nanoparticles for drug and gene delivery. ACS Nano, 4:1049-1059, 2010.

[2] R. Ravichandran, S. Sundarrajan, J. R. Venugopal, S. Mukheriee, and S. Ramakrishna. Applications of conducting polymers and their issues in biomedical engineering. J. R. Soc. Interface, 7:S559-S579, 2010.

[3] D. F. Moyano and V. M. Rotello. Nano meets biology: Structure and function at the nanoparticle interface. Langmuir, 27:10376-10385, 2011.

[4] X. Zhuang, Y. Mai, D. Wu, F. Zhang, and X. Feng. Two dimensional soft nanomaterials: a fascinating world of materials. Adv. Mat., 27:403-427, 2015.

[5] P. Wang, Y. Wang, and L. Tong. Functionalized polymer nanofibers: a versatile platform for manipulating light at the nanoscale. Light: Science and Applications, 2:102-112, 2013.

[6] L. M. Bronstein, V. G. Matveeva, and E. M. Sulman. Nanoparticulate catalysts based on nanostructured polymers. Wiley-VCH, 2008.

[7] J. Peet, A. J. Heeger, and G. C. Bazan. Plastic solar cells: self assembly of bulk heterojunction nanomaterials by spontaneous phase separation. Acc. Chem. Res., 42:1700-1708, 2009.

[8] I. Fratoddi, P. Altamura, A. Bearzotti, A. Furlani, and M. V. Russo. Electrical and morphological characterization of poly(nonosubstituted )acetylene based membrane: applications as humidity and organic vapor sensors. Thin Solid Films, 458:292-298, 2004.

[9] I. Fratoddi, C. Battocchio, A. L. Groia, and M. V. Russo. Nanostructured polymetallaynes of controlled length: synthesis and characterization of oligomers and polymers from 1,1'-bis- (ethynyl) 4,4' -biphenyl bridging pt(ii) or pd(ii) centers. J. Polym. Sci. Part A, Polym. Chem., 45:33113329, 2007.

[10] C. Battocchio, I. Fratoddi, M. V. Russo, and G. Polzonetti. Nexafs study of a pt-containing rod-like organometallic polymer (pt-debp): molecular orientation onto hopg au/si(111), $\mathrm{cr} / \mathrm{si}(111)$ and si(111) surfaces. Chem. Phys. Lett., 400:290-295, 2004.

[11] S. Guo and E. Wang. Functional micro/nanostructures: Simple synthesis and application in sensors, fuel cells, and gene delivery. Accounts of Chemical Research, 44:491-500, 2011. 
[12] I. Fratoddi, I. Venditti, C. Battocchio, G. Polzonetti, F. Bondino, M. Malvestuto, E. Piscopiello, L. Tapfer, and M. V. Russo. Gold nanoparticles dyads stabilized with binuclear pt(ii) dithyol gridges. J. Phys. Chem. C, 115:15198-15204, 2011.

[13] C. Caliendo, G. Contini, I. Fratoddi, S. Irrera, P. Pertici, G. Scavia, and M. V. Russo. Nanostructured organometallic polymer and palladium/polymer hybrid: surface investigation and sensitivity to relative humidity and hydrogen in surface acustic wave sensors. Nanotechnology, 18:125504-7pp, 2007.

[14] L. Chronopoulou, I. Fratoddi, C. Palocci, I. Venditti, and M. V. Russo. Osmosis based method drives the self-assembly of polymeric chains into micro- and nanostructures. Langmuir, 25:11940-11946, 2009.

[15] I. Fratoddi, I. Venditti, and M. V. Russo. Advances in macromolecules: perspectives and applications. Springer, 2010.

[16] J. P. Rau and K. E. Geckeler. Polymer nanoparticles: preparation techniques and size control parameters. Prog. Polym. Sci., 36:887-913, 2011.

[17] R. D'Amato, I. Venditti, M. V. Russo, and M. Falconieri. Growth control and long-range self-assembly of poly(methyl methacrylate) nanospheres. J. Appl. Polym. Sci., 102:4493-4499, 2006.

[18] I. Venditti, R. D'Amato, M. V. Russo, and M. Falconieri. Synthesis of conjugated polymeric nanobeads for photonic bandgap materials. Sensors Act. B, 126:35-40, 2007.

[19] I. Venditti, I. Fratoddi, C. Battocchio, G. Polzonetti, C. Cametti, and M. V. Russo. Soluble polymers of monosubstituted acetylenes with quaternary ammonium pendant groups: structure and morphology. Polymer International, 60:1222-1229, 2011.

[20] S. Schutzmann, P. Prosposito, M. Casalboni, I. Venditti, and M. V. Russo. Spectroscopic ellipsometry on photonic crystals made by selfassembled dye-doped p(s/hema) nanospheres. Physica Status Solidi C, 5:1403-1406, 2008.

[21] R. De Angelis, I. Fratoddi, F. De Matteis, P. Prosposito, I. Cacciotti, L. D'Amico, F. Nanni, A. Yadav, M. Casalboni, and M. V. Russo. From nanospheres to microribbons: self-assembled eosin y doped pmma nanoparticles as photonic crystals. J. Colloid Interface Sci., 414:24-32, 2014.

[22] C. Palocci, L. Chronopoulou, I. Venditti, E. Cernia, M. Diociauti, I. Fratoddi, and M. V. Russo. Lipolytic enzymes with improved activity and selectivity upon adsorption on polymeric nanoparticles. Biomacromol., 8:3047-3053, 2007. 
[23] I. Venditti, I. Fratoddi, M. V. Russo, S. Bellucci, R. Crescenzo, L. Iozzino, M. Staiano, V. Aurilia, A. Varriale, M. Rossi, and S. DAuria. Nanobeadsbased assays. the case of gluten detection. J. Phys.: Condens. Matter, 20:474202-474205, 2008.

[24] M. Bassetti, I. Fratoddi, L. Lilla, C. Pasquini, M. V. Russo, and O. Ursini. Synthesis of polyarylacetylenes by -ray-induced polymerization of terminal alkynes. nanostructures of ortho-substituted derivatives. J. Polymer Sci., Part A: Polym. Chem., 50:5097-5106, 2012.

[25] A. Eftekhari. Nanostructured Conductive Polymers. John Wiley and Sons, 2011.

[26] f. Rajesha, A. Tarushee, and K. Devendra. Recent progress in the development of nano-structured conducting polymers/nanocomposites for sensor applications. Sensors and Actuators B, 136:275-286, 2009.

[27] J. Wang and D. Zhang. One dimensional nanostructured polyanyline: synthesis, morphology, controlling, formation mechanisms, new features and applications (review). Adv. Polym. Techn., 32:E323-E368, 2013.

[28] M. Joulazadeh, A. H. Navarchian, and M. Niroomand. A compararive study on humidity sensing performances of polyaniline and polypyrrole nanostructures (article). Adv. Polym. Techn., 33:21461-11pp, 2014.

[29] V. C. Gonalves and D. T. Balogh. Optical chemical sensors using polythiophene derivatives as active layer for detection of volatile organic compounds. Sensors and Actuators B, 162:307-312, 2012.

[30] R. D'Amato, L. Medei, I. Venditti, and V. M. Russo. Chemical synthesis of polyphenylacetylene nanospheres with controlled dimensions for photonic crystals. Mat. Sci. Eng. C, 23:861-865, 2003.

[31] I. Fratoddi, N. Panziera, P. Pertici, G. Martra, L. Bertinetti, and M. V. Russo. Nanostructured gold/conjugated polymer hybrids: Preparation, chemical structure and morphology. Mat. Sci. Engin. C, 27:1305-1308, 2007.

[32] F. Vitale, L. Mirenghi, E. Piscopiello, G. Pellegrini, E. Trave, G. Mattei, I. Fratoddi, M. V. Russo, L. Tapfer, and P. Mazzoldi. Gold nanoclustersorganometallic polymer nanocomposite: synthesis and characterization. Mat. Sci. Engin. C, 27:1300-1304, 2007.

[33] D. W. Hatchett and M. Josowicz. Composites of intrinsically conducting polymers as sensing nanomaterials. Chem. Rev., 108:746-769, 2008.

[34] X. Lua, W. Zhang, C. Wang, T. C. Wen, and Y. Wei. One-dimensional conducting polymer nanocomposites: Synthesis, properties and applications. Progress in Polymer Science, 36:671-712, 2011. 
[35] E. Traversa. Ceramic sensors for humidity detection: the state-of-the-art and future developmentsators. Sens. Actuators, B, 23:135-156, 1995.

[36] H. Arai and T. Seyama. Sensors: a comprehensive survey, chemical and biochemical sensors. Wiley, London, 1991.

[37] N. A. Luechinger, S. Lecher, E. K. Athanassion, R. N. Grass, and W. J. Stark. Highly sensitive optical detection of humidity on polymer/metal nanoparticle hybrid films. Langmuir, 23:3473-3477, 2007.

[38] E. Zampetti, S. Pantalei, A. Pecora, A. Valletta, N. Maioli, A. Mintoti, A. Macagnano, G. Fortunato, and A. Bearzotti. Design and optimization of an ultra thin flexible capacitive humidity sensor. Sensors and Actuators B, 143:302-307, 2009.

[39] Y. Sakai, Y. Sadaoka, and M. Matsuguchi. Humidity sensors based on polymer thin films. Sensors Actuators B, 35:85-90, 1996.

[40] C. Cantalini and M. Perlino. Microstructure and humidity-sensituve characteristics of a iron (iii) oxide ceramic sensor. J. Am. Ceram. Soc., 75:546-551, 1992.

[41] P. J. Su and M. Y. Tsai. Humidity sensing and electrical properties of a composite material of nano-sized $\mathrm{sio}_{2}$ and poly(2- acrylamido -2methylpropane sulfonate. Sensors and Actuators B, 100:417-422, 2004.

[42] R. J. Wu, C. C. Lin, H. W. Chen, and M. Chavali. Composite of $\mathrm{tio}_{2}$ nanowires and nafion as humidity sensor materials. Sensors and Actuators B, 115:198-204, 2006.

[43] A.G. Foster. The sorption of condensible vapours by porous solids. part i. the applicability of the capillary theory. Trans. Faraday Soc., 28:645-657, 1932.

[44] B. Yang, B. Aksak, Q. Lin, and M. Sitti. Compliant and low-cost humidity nanosensors using nanoporous polymer membranes. Sensors Actuators B, 114:254-262, 2006.

[45] Y. Wang and J. T. W. Yeow. Humidity sensing of ordered microporous silica with $\mathrm{hfo}_{2}$ thin film coating. IEEE Sens. J., 9:541-547, 2009.

[46] R. Hilfer. Transport and relaxation phenomena in porous media. Adv. Chem. Phys., 92:299-424, 1996.

[47] S. Stolzle, A. Enders, and G. Nimtz. Numerical simulation of random composite dielectrics. J. Phys. I, 2:401-408, 1992.

[48] R. Pelster. Dielectric spectroscopy of confinement effects in polar materials. Phys. Rev. B, 59:9214-9228, 1999. 
[49] Y. N. Kunishima, N. Miyayama, and H. Yanagida. Effects of the external electric field from a substrate on cl2 gas adsorption on sno2 thin films. Appl. Phys. Lett., 69:632-634, 1996.

[50] D. S. Mclachlan. The complex permittivity of emulsions: an effective media-percolation equation. Solid State Commun., 72:831-834, 1989.

[51] Y. Wang, S. Park, J. T. W. Yeow, A. Langner, and F. Muller. A capacitive humidity sensor based on ordered macroporous silicon with thin film surface coating. Sensors and Actuators B, 149:136-142, 2010.

[52] J. Das, S. M. Hossain, and S. Chahraborty. Role of parasitic in humidity sensing by porous silicon. Sensors Actuators A, 94:44-52, 2001.

[53] D. J. Bergman. The dielectric constant of a composite material: a problem in classical physics. Phys. Rep., 43:377-407, 1978.

[54] C. J. F. Bottcher and P. Bordewijk. Theory of electric polarization. Elsevier, N.Y., 1978.

[55] R. G. Geyer, J. Baker-Jarvis, T. A. Vanderah, and J. Manese. Advances in dielectric ceramic materials. Am. Ceramic Soc., Wasterville, Ohio, 1998.

[56] E. Haslund, B. D. Hansen, R. Hilfer, and B. Nost. Measurement of local porosities and dielectric dispersion for water-saturated porous medium. J. Appl. Phys., 76:5473-5480, 1994.

[57] R. Hilfer. Geometric and dielectric characterization of porous media. Phys. Rev. B, 44:60-75, 1991.

[58] A. Hilfer, T. Rage, and B. Virgin. Local percolation probabilities for a natural sandstone. Physica A, 241:105-110, 1997.

[59] J. Widjajakusuma, C. Manwart, B. Biswal, and R. Hilfer. Quantitative comparison of mean field moxing laws for conductivity and dielectric constants of vporous media. Physica A, 270:325-331, 1999.

[60] R. Hilfer. Local porosity theory for electrical and hydrodynamical transport through porous media. Physica A, 194:406-414, 1993.

[61] M. Clausse. Encyclopedia of emulsion technology: basic theory. M. Dekker, New York, 1983.

[62] R. Hilfer. Local-porosity theory for flow in porous media. Phys. Rev. B, 45:7115-7121, 1992.

[63] K. Nitsch, B. W. Licznerski, H. Teterycz, L. J. Golonka, and K. Wisniewski. Ac equivalent circuits of thick film humidity sensors. Vacuum, 50:131-137, 1998. 
[64] J. Wang, Q. H. Lin, R. Q. Zhou, and B. K. Xu. Humidity sensors based on composite material of nano-batio3 and polymer rmx. Sensors and Actuators B, 81:248-253, 2002.

[65] T. Zhang, Y. He, R. Wang, W. Geng, L. Wang, L. Niu, and X. Li. Analysis of dc and ac properties of humidity sensors based on polypyrrole materials. Sensors and Actuators, 131:687-691, 2008.

[66] M. J. Yang, T. Li, N. Camaioni, G. Casalbore-Miceli, A. Martelli, and G. Ridolfi. Polymer electrolytes as humidity sensors: progress in improving an impedance device. Sensors and Actuators B, 86:229-234, 2002.

[67] G. Casalbore-Miceli, A. Zanelli, E. M. Girotto, M. J. Yang, Y. S. Chen, and Y. Li. A model of polyelectrolyte conductance in moist solvents as a basis of water sensors. Langmuir, 21:9704-9708, 2005.

[68] G. Casalbore-Miceli, A. Zanelli, A. W. Rinaldi, N. Camaioni, M. J. Yang, Y. Li, and E. M. Girotto. Electric properties of polyelectrolyte films in moist solvents. Sensors and Actuators B, 125:120-125, 2007.

[69] H. J. Liou, G. Suyambrakasam, T. C. Tsai, R. J. Wu, and M. Chavali. Preparation of nanostructured organic/inorganic polymer hybrids and their humidity sensing properties. J. Taiwan Inst. Chem. Eng., 42:868873, 2011.

[70] Q. Lin and M. Li. Investigations on the sensing mechanism of humidity sensors based on electrospun polymer nanofibers. Sensors and Actuators $B, 171 / 172: 309-314,2012$.

[71] K. K. Kanazawa and J. G. Gordon. Frequency of a quartz microbalance in conctact with a liquid. Anal. Chem., 57:1770-1771, 1985.

[72] I. Venditti, A. Bearzotti, A. Macagnano, and M. V. Russo. Enhanced sensitivity of ppa and ppahema nanobeads to humidity. Sensor Lett., $5: 1-5,2007$.

[73] K. Jaruwongrungsee, A. Tuantranont, Y. Wanna, A. Wisitsoraat, and T. Lomas. Quartz crystal microbalance humidity sensor using electrospun pani micro/nano dots. Nanotechnology, pages 316-319, 2007.

[74] P. Story, D. Galipeau, and R. D. Mieham. A study of low-cost sensors for measuring low relative humidity. Sensors and Actuators B, 24/25:681685, 1995.

[75] A. R. K. Ralston, C. F. Klein, P. E. Thoma, and D. D. Denton. A model for the relative environmental stability of a series of polyimide capacitance humidity sensors. Sensors and Actuators B, 34:343-348, 1996. 
[76] M. Matsuguchi, T. Kuroiwa, T. Miyagishi, S. Suzuki, T. Ogura, and Y. Sakai. Stability and reliability of capacitive-type relative humidity sensors using crosslinked polyimide films. Sensors and Actuators B, 52:53$57,1998$.

[77] P. M. Harrey, B. J. Ramsey, P. S. A. Evans, and D. J. Harrison. Capacitive-type humidity sensors fabricated using the offset lithographic printing process. Sensors and Actuators B, 87:226-232, 2002.

[78] Y. Sakai, M. Matsuguchi, and T. Hurukawa. Humidity sensor using crosslinked poly(chloromethyl styrene). Sens. Actuators, B, 66:135-138, 2000.

[79] C. Roman, O. Bodea, N. Prodan, A. Levi, E. Cordos, and I. Manoviciu. A capacitive-type humidity sensor using crosslinked poly(methylmethacrylate-co-(2 hydroxypropyl)-methacrylate). Sensors and Actuators B, 24/25:710-713, 1995.

[80] A. R. K. Ralston, J. A. Tobin, S. S. Bajikar, and D. Denton. Comparative performance of linear, cross-linked, and plasma-deposited pmma capacitive humidity sensors. Sensors and Actuators B, 22:139-147, 1994.

[81] K. Suzuki, Y. Mimura, Y. Ishizaka, Y. Nabeta, and T. Inuzuka. A humidity sensor with linear relationship in relative humidity-electrical capacitance characteristics. Proc. Tech. Digest 12th Sensor Symposium, pages 129-132, 1994.

[82] T. Kuroiwa, T. Miyagishi, A. Ito, M. Matsuguchi, Y. Sadaoka, and Y. Sakai. A thin-film polysufone-based capacitive-type relative-humidity sensor. Sensors and Actuators B, 24/25:692-695, 1995.

[83] V. Ducere, A. Bernes, and C. Lacabanne. A capacitive humidity sensor using cross-linked cellulose acetate butyrate. Proc. Techn. Digest of the 12th Sensor Symposium, pages 129-132, 1994.

[84] K. P. Yoo, L. T. Lim, M. J. Lee, C. J. Lee, and C. W. Park. Novel resistive.type humidity sensor based on multiwall carbon nanotube-polymide composite film. Sensors Actuators B, 145:120-125, 2010.

[85] Q. Lin, W. Li, and M. Yang. Polyaniline nanofiber humidity sensor prepared by electrospinning. Sensors Actuators B, 161:967-972, 2012.

[86] P. Li, Y. Li, and M. Yang. Electrospun nanofibers of polymer composite as promising humidity sensitive material. Sens. Actuators, B, 141:390$395,2009$.

[87] X. Wang, B. Ding, J. Yu, and M. Wang. Highly sensitive humidity sensors based on electro-spinning/netting a polyamide 6 nano-fiber/net modified by polyethyleneimine. J. Mater. Chem., 21:16231-16238, 2011. 
[88] A. Sun, Y. Wang, and Y. Li. Stability and water-resistance of humidity sensors using crosslinked and quaternized polyelectrolytes films. Sens. Actuators, B, 145:680-684, 2010.

[89] S. Tsuchitani, T. Sugawara, N. Kinjo, S. Ohara, and T. Tsunoda. Humidity sensor using ionic copolymer and it application to humiditytemperature sensor module. Sens. Actuators, 15:375-386, 1988.

[90] K. L. Rauen, D. A. Smith, W. R. Heineman, J. Johnson, R. Seguin, and P. Stoughton. Humidity sensor based on conductivity measurements of poly(dimethyil diallyl ammonium chloride) polymer film. Sens. Actuators $B, 17: 61-68,1993$.

[91] Y. Sakai, M. Matsuguchi, T. Hurukawa, and M.J. Tamura. A humidity sensor using polytetrafluoroethylene-graft-quaternizedpolyvinylpyridine. J. Electrochem. Soc., 138:2474-2478, 1991.

[92] Y. Sakai, M. Matsuguchi, Y. Sadaoka, and K. Hirayama. A humidity sensor composed of interpenetrating polymer networks of hydrophilic and hydrophobic methacrylate polymers. J. Electrochem. Soc., 140:432-436, 1993.

[93] M. S. Gong, M. H. Li, and H. W. Rhee. Humidity sensor using cross-linked copolymers containing viologen moiety. Sens. Actuators, B, 73:185-191, 2001.

[94] S. H. Park, J. S. Park, C. W. Lee, and M. S. Gong. Humidity sensor using gel polyelectrolyte prepared from mutually reactive copolymers. Sens. Actuators, B, 86:68-74, 2002.

[95] S. S. Lee and M. S. Gong. Humidity sensitive properties of humidity sensor using quaternized cross-linked copolymers of vinylbenzyl chloride. Korean J. Mater. Res., 10:825-830, 2000.

[96] C. W. Lee, S. W. Joo, and M. S. Gong. Polymeric humidity sensor using polyelectrolytes derived from alkoxysilane cross-linker. Sensors and Actuators B, 105:150-158, 2005.

[97] Y. S. Chen, Y. Li, and M. J. Yang. A fast response resistive thin film humidity sensor based on poly(4-vinylpyridine) and poly(glycidyl methacrylate). J. Appl. Polym. Sci., 105:3470-3475, 2007.

[98] Y. Sakai, M. Matsuguchi, and T. Hurukawa. Humidity sensor using crosslinked poly(chloromethyl styrene). Sens. Actuators B, 66:135-138, 2000.

[99] S. Wu, Y. Zhu, F. Li, and J. Shen. Humidity sensing properties of the vinylpyridine-butyl acrylate-styrene copolymers. J. Appl. Polym. Sci., 74:1992-1996, 1999. 
[100] G. Casalbore-Miceli, M. J. Yang, Y. Li, A. Zanelli, A. Martelli, S. Chen, and N. A. She, Y.and Camaioni. Polyelectrolyte as humidity sensing material: Influence of the preparation parameters on its sensing property. Sens. Actuators B, 114:584-590, 2006.

[101] T. Fei, H. Zhas, K. Jiang, and T. Zhang. Synthesis and humidity sensitive properties of cross-linked water resistant polymer electrolytes. Sens. Actuators B, 208:277-282, 2015.

[102] T. Fei, H. Zhas, K. Jiang, and T. Zhang. Polymeric humidity sensors with nonlinear response: Properties and mechanism investigation. $J$. Appl. Polymer Sci., 130:2056-2061, 2013.

[103] E. Kim, S. Y. Kim, G. Jo, S. Kim, and M. J. Park. Colorimetric and resistive polymer electrolyte thin films for realtime humidity sensors. ACS Appl. Mater. Interfaces, 4:5179-5187, 2012.

[104] X. Lv, Y. Li, R. Li, and M. Yang. A resistive-type humidity sensor based on cross-linked polyelectrolytes prepared by uv irradiation. Sensors and Actuators, 135:581-586, 2009.

[105] P. Pattananuwat, M. Tagaya, and T. Kobayashi. A novel highly sensitive humidity sensor based on poly(pyrrole -co- pyrrole) copolymer film: Ac and dc impedance analysis. Sensors and Actuators B, 209:186-193, 2015.

[106] J. Liu, M. Agarwal, K. Varahramyan, E.S. Berney, and W.D. Hodo. Polymer-based microsensor for soil moisture measurement. Sensors and Actuators B, 129:599-604, 2008.

[107] P.J. Skabara, L. Li, F. Vilela, D. Uttamchandani, and J. Forgie. Miniature humidity micro-sensor based on organic conductive polymerpoly $(3,4-$ ethylenedioxythiophene). Micro Nano Lett., 4:84-87, 2009.

[108] Y. Miyoshi, K. Miyajima, H. Saito, H. Kudo, T. Takeuchi, I. Karube, and K. Mitsubayashi. Humidity sensor in a sandwich configuration with a hydrophilic porous membrane. Sens. Actuators B, 142:28-32, 2009.

[109] Z. Chen and C. Lu. Humidity sensors: a review of materials and mechanisms. Sensor Lett., 3:274-295, 2005.

[110] H. Grange, C. Beith, H. Boucher, and G. Delapierre. A capacitive humidity sensor with every fast response time and very low hysteresis. Sensors and Actuators, 12:291-296, 1987.

[111] M. Matsuguchi, S. Kubo, and Y. Sakai. Humidity sensors. Electrochemistry, 67:170-178, 1999.

[112] M. Matsuguchi, Y. Sadaoka, Y. Nuwa, M. Shinmoto, Y. Sakai, and T. Kuroiwa. Capacitive-type humidity sensors using polymerized vinyl carboxylate. J. Electrochem. Soc., 141:614-618, 1994. 
[113] J. Perez and C. Freyre. A poly(ethylene terephthalate)-based humidity sensor. Sensors and Actuators B, 42:27-30, 1997. 